ORIGINAL ARTICLE

\section{Genetic PTX3 Deficiency and Aspergillosis in Stem-Cell Transplantation}

\author{
Cristina Cunha, Ph.D., Franco Aversa, M.D., João F. Lacerda, M.D., Ph.D., \\ Alessandro Busca, M.D., Oliver Kurzai, M.D., Matthias Grube, M.D., \\ Jürgen Löffler, Ph.D., Johan A. Maertens, M.D., Ph.D., Alain S. Bell, Ph.D., \\ Antonio Inforzato, Ph.D., Elisa Barbati, Ph.D., Bruno Almeida, Ph.D., \\ Pedro Santos e Sousa, M.D., Anna Barbui, M.D., Leonardo Potenza, M.D., Ph.D., \\ Morena Caira, M.D., Ph.D., Fernando Rodrigues, Ph.D., Giovanni Salvatori, Ph.D., \\ Livio Pagano, M.D., Mario Luppi, M.D., Ph.D., Alberto Mantovani, M.D., \\ Andrea Velardi, M.D., Luigina Romani, M.D., Ph.D., and Agostinho Carvalho, Ph.D.
}

A BSTRACT

BACKGROUND

The soluble pattern-recognition receptor known as long pentraxin 3 (PTX3) has a nonredundant role in antifungal immunity. The contribution of single-nucleotide polymorphisms (SNPs) in PTX3 to the development of invasive aspergillosis is unknown.

\section{METHODS}

We screened an initial cohort of 268 patients undergoing hematopoietic stem-cell transplantation (HSCT) and their donors for PTX3 SNPs modifying the risk of invasive aspergillosis. The analysis was also performed in a multicenter study involving 107 patients with invasive aspergillosis and 223 matched controls. The functional consequences of PTX3 SNPs were investigated in vitro and in lung specimens from transplant recipients.

\section{RESULTS}

Receipt of a transplant from a donor with a homozygous haplotype (h2/h2) in PTX3 was associated with an increased risk of infection, in both the discovery study (cumulative incidence, $37 \%$ vs. $15 \%$; adjusted hazard ratio, 3.08; $\mathrm{P}=0.003$ ) and the confirmation study (adjusted odds ratio, 2.78; $\mathrm{P}=0.03$ ), as well as with defective expression of PTX3. Functionally, PTX3 deficiency in h2/h2 neutrophils, presumably due to messenger RNA instability, led to impaired phagocytosis and clearance of the fungus.

\section{CONCLUSIONS}

Genetic deficiency of PTX3 affects the antifungal capacity of neutrophils and may contribute to the risk of invasive aspergillosis in patients treated with HSCT. (Funded by the European Society of Clinical Microbiology and Infectious Diseases and others.)
The authors' affiliations are listed in the Appendix. Address reprint requests to Dr. Carvalho at the Department of Experimental Medicine and Biochemical Sciences, University of Perugia, Polo Unico Sant'Andrea delle Fratte, 06132 Perugia, Italy, or at agostinho.carvalho@ unipg.it.

N Engl J Med 2014;370:421-32. DOI: 10.1056/NEJMoal211161

Copyright $\odot 2014$ Massachusetts Medical Society. 
ONG PENTRAXIN 3 (PTX3) IS A SOLUBLE pattern-recognition receptor produced by phagocytes and nonimmune cells at sites of inflammation or injury. In addition to its major role in female fertility and vascular biology, ${ }^{1}$ PTX3 has a nonredundant role in modulating various effector pathways involved in immune resistance to Aspergillus fumigatus, including activating innate immune cells ${ }^{2}$ and driving protective adaptive immunity. ${ }^{3}$ PTX3 forms complexes on the conidial surface of the fungus and acts as an opsonin, enhancing recognition and phagocytosis of conidia through mechanisms that depend on Fc $\gamma$ receptor, CD11b, and complement. ${ }^{4}$ The interaction of PTX3 with the yeast phase of Candida albicans ${ }^{5}$ and Paracoccidioides brasiliensis ${ }^{6}$ has also been reported.

Mononuclear phagocytes and dendritic cells synthesize PTX3 de novo in response to microbial moieties or inflammatory signals. In contrast, PTX3 is stored in a ready-made form in granules from mature polymorphonuclear leukocytes, and its secretion and localization to extracellular traps promote the control of A. fumigatus infection in vivo. ${ }^{7}$ Therefore, it is not surprising that Ptx3 deficiency renders mice susceptible to pulmonary aspergillosis because of the defective recognition of conidia by neutrophils, alveolar macrophages, and dendritic cells. ${ }^{3,4}$ The susceptibility phenotype is associated with impaired activation of a protective type 1 helper T-cell (Th1) antifungal response coupled with a detrimental type 2 helper T-cell response. ${ }^{3}$ The administration of exogenous Ptx3 in mice that have undergone bone marrow transplantation reverts the susceptibility to disease by restoring the Th1 response $^{3}$; the administration of exogenous Ptx3 also restrains the type 17 helper T-cell response and associated pathogenic inflammation induced during infection in experimental models of chronic granulomatous disease. ${ }^{8}$ The protective effect of exogenous Ptx3 is further substantiated by the capacity of the protein to improve the efficacy of antifungal therapy in mice when the two treatments are administered in combination. ${ }^{9,10}$

The pivotal role of PTX3 in innate antifungal immunity ${ }^{11}$ makes the protein an attractive candidate for studies of genetic susceptibility to fungal diseases in high-risk patients, such as those undergoing hematopoietic stem-cell transplantation (HSCT). Invasive aspergillosis may develop as a complication in 5 to $15 \%$ of patients undergoing allogeneic HSCT. ${ }^{12-14}$ We screened patients undergoing HSCT and their donors for single-nucleotide polymorphisms (SNPs) in the PTX3 gene and assessed the association of identified SNPs with susceptibility to invasive aspergillosis and impaired innate antifungal immunity.

\section{METHODS}

\section{PATIENTS AND STUDY PROCEDURES}

All adult patients with hematologic disorders who were undergoing allogeneic HSCT at the Hospital of the University of Perugia, in Perugia, Italy, between 2003 and 2011, and their respective donors (who were related in $98 \%$ of cases), were eligible for the discovery study. Among 430 transplantations performed in this period, 346 recipientdonor pairs with available DNA and patient-level data were identified. Exclusion criteria were participation in clinical studies (64 patients), ${ }^{15}$ infection with invasive molds other than aspergillus species (12), and pretransplantation infection with molds (2). Fifty-one cases of invasive aspergillosis were classified as "probable" (in 26 patients) or "proven" (in 25 patients), according to the revised standard criteria from the European Organization for Research and Treatment of Cancer-Mycoses Study Group ${ }^{16}$ (Table 1). Among these cases, 9 (18\%) occurred in the neutropenic period and 42 (82\%) after engraftment. Transplantation procedures were performed as previously described. ${ }^{17}$ Graft-versus-host disease (GVHD) and cytomegalovirus disease were diagnosed according to standard criteria. ${ }^{18,19}$ Conditioning regimens and prophylaxis are described in the Supplementary Appendix, available with the full text of this article at NEJM.org. The study was approved by the Umbria Regional Hospital Ethics Committee in Perugia, and patients and donors provided informed written consent for data collection, DNA and cell storage, and their use for diagnostic and research purposes.

The methods used for the confirmation study (Table S1 in the Supplementary Appendix) and the genetic and functional analyses are detailed in the Supplementary Appendix.

\section{STATISTICAL ANALYSIS}

In the analysis of the discovery data set, the probability of invasive aspergillosis according to PTX3 variants was determined with the use of the 


\begin{tabular}{|c|c|c|c|}
\hline Clinical Variable & $\begin{array}{c}\text { Invasive } \\
\text { Aspergillosis } \\
(\mathrm{N}=51)\end{array}$ & $\begin{array}{c}\text { No Invasive } \\
\text { Aspergillosis } \\
(\mathrm{N}=217)\end{array}$ & P Value \\
\hline \multicolumn{4}{|l|}{ Age at transplantation — no. (\%) } \\
\hline$<40 \mathrm{yr}$ & $26(51)$ & $112(52)$ & \\
\hline$\geq 40 \mathrm{yr}$ & $25(49)$ & $105(48)$ & 1.00 \\
\hline \multicolumn{4}{|l|}{ Sex - no. (\%) } \\
\hline Male & $23(45)$ & $107(49)$ & \\
\hline Female & $28(55)$ & $110(51)$ & 0.64 \\
\hline \multicolumn{4}{|l|}{ Sex of donor-recipient pair — no. (\%) } \\
\hline Female-male & $10(20)$ & $58(27)$ & \\
\hline Other & $41(80)$ & $159(73)$ & 0.37 \\
\hline \multicolumn{4}{|l|}{ Transplantation type - no. (\%) } \\
\hline Transplant from HLA-matched related donor & $14(27)$ & $90(42)$ & \\
\hline Transplant from HLA-mismatched related donort & $37(73)$ & $122(56)$ & 0.06 \\
\hline Transplant from HLA-matched unrelated donor & 0 & $5(2)$ & 0.62 \\
\hline Transplant from HLA-mismatched unrelated donor & 0 & 0 & 1.00 \\
\hline \multicolumn{4}{|l|}{ Underlying disease - no. (\%) } \\
\hline Acute leukemia & $31(61)$ & $152(70)$ & \\
\hline Lymphoma or myeloma & $14(27)$ & $53(24)$ & 0.58 \\
\hline Chronic leukemia & $4(8)$ & $11(5)$ & 0.48 \\
\hline Other & $2(4)$ & $1(1)$ & 0.08 \\
\hline \multicolumn{4}{|l|}{ Disease stage — no. (\%) } \\
\hline First complete remission & $14(27)$ & $73(34)$ & \\
\hline Second or subsequent remission, or relapse & $37(73)$ & $144(66)$ & 0.41 \\
\hline \multicolumn{4}{|l|}{ Myeloablative conditioning regimen — no. (\%) } \\
\hline Total-body irradiation & $43(84)$ & $150(69)$ & \\
\hline No total-body irradiation & $8(16)$ & $67(31)$ & 0.04 \\
\hline \multicolumn{4}{|l|}{ CMV serostatus of donor and recipient - no. (\%) } \\
\hline $\begin{array}{l}\text { Donor and recipient CMV-negative or donor CMV-positive } \\
\text { and recipient CMV-negative }\end{array}$ & $5(10)$ & $27(12)$ & \\
\hline $\begin{array}{l}\text { Donor CMV-negative and recipient CMV-positive or donor } \\
\text { and recipient CMV-positive }\end{array}$ & $46(90)$ & $190(88)$ & 0.65 \\
\hline Duration of neutropenia - median no. of days (range) $\mathbb{R}$ & $16(10-24)$ & $16(10-40)$ & 0.39 \\
\hline Acute GVHD, grade II, III, or IV — no. (\%) & $5(10)$ & $10(5)$ & 0.17 \\
\hline \multicolumn{4}{|l|}{ Antifungal prophylaxis — no. (\%)ף } \\
\hline Liposomal amphotericin B & $49(96)$ & $177(82)$ & \\
\hline Fluconazole & $2(4)$ & $40(18)$ & 0.02 \\
\hline
\end{tabular}

* CMV denotes cytomegalovirus, and GVHD graft-versus-host disease.

$\dagger P$ values were calculated by Fisher's exact probability t-test, or by Student's t-test for continuous variables.

Recipients of transplants from mismatched related donors received rabbit antithymocyte globulin as part of their pretransplantation conditioning regimen.

$\int$ Neutropenia was defined as $0.1 \times 10^{9}$ neutrophils per liter or less.

9 Antifungal prophylaxis consisted of fluconazole for standard-risk patients and liposomal amphotericin B for high-risk patients. At the time of allogeneic hematopoietic stem-cell transplantation, patients were considered to be at high risk for invasive fungal disease if they were receiving a transplant from an HLA-mismatched related donor or if they were receiving a transplant from an HLA-identical sibling and had undergone conditioning that included total-body irradiation. Patients were considered to be at standard risk if they were receiving a transplant from an HLA-identical sibling and had not undergone conditioning with total-body irradiation. 
cumulative incidence method, and PTX3 status among patients with and those without infection was compared with the use of Gray's test. ${ }^{20} \mathrm{Cu}-$ mulative incidences were computed with $\mathrm{R}$ software, version 2.10 .1 (cmprsk package), ${ }^{21}$ with censoring of data at the date of the last follow-up visit, and with relapse and death as competing risks. A period of 24 months was chosen to include all cases in the discovery cohort. Overall survival, defined as the time from transplantation to death from any cause, was estimated with the use of the Kaplan-Meier method and assessed according to PTX3 status with the use of the logrank test. Additional details on statistical methods are provided in the Supplementary Appendix.

\section{RESULTS}

\section{GENETIC VARIANTS IN DONOR PTX3 AND THE RISK OF INVASIVE ASPERGILLOSIS}

The PTX3 gene was analyzed for 22 SNPs spanning approximately $25 \mathrm{~kb}$ on chromosome 3 (Table S2 in the Supplementary Appendix). We assessed the risk of invasive aspergillosis according to recipient or donor PTX3 genotypes by estimating the cumulative incidence of infection among transplant recipients 24 months after transplantation. Several SNPs in the donors were associated with a risk of invasive aspergillosis in the recipients. We did not observe an association between SNPs in recipients and a risk of infection (Table S3 in the Supplementary Appendix). Among these SNPs, only $+281 \mathrm{~A} / \mathrm{G},+734 \mathrm{~A} / \mathrm{C}$ (D48A), and $+1449 \mathrm{~A} / \mathrm{G}$ remained significantly associated with invasive aspergillosis after correction for multiple testing. The cumulative incidence of invasive aspergillosis among patients with $+281 \mathrm{~A} / \mathrm{G}$ and $+1449 \mathrm{~A} / \mathrm{G}$ was $37 \%$ for genotype GG and $14 \%$ for genotypes AA and AG combined $(\mathrm{P}<0.001$; adjusted $\mathrm{P}=0.002$ ) (Fig. $1 \mathrm{~A}$ ); the cumulative incidence of invasive aspergillosis among patients with $+734 \mathrm{~A} / \mathrm{C}$ was $34 \%$ for genotype AA and $14 \%$ for genotypes $\mathrm{AC}$ and $\mathrm{CC}$ combined $(\mathrm{P}<0.001$; adjusted $\mathrm{P}=0.005$ ) (Fig. 1B).

Analysis of linkage disequilibrium between markers showed the existence of three distinct linkage-disequilibrium blocks (Fig. S1 in the Supplementary Appendix). Haplotype analysis revealed that an absence of donor haplotypes T-A (block 1), A-A (block 2), and A-C (block 3) was associated with an increased risk of infection
Figure 1 (facing page). Cumulative Incidence of Invasive Aspergillosis and Estimated Overall Survival after Hematopoietic Stem-Cell Transplantation in 268 Patients in the Discovery Cohort, According to Donor PTX3 Variants.

Panels $A, B, C$, and $D$ show the probability of proven or probable invasive aspergillosis, with censoring of data at 24 months and with relapse and death as competing events. P values were calculated with the use of Gray's test. Panel E shows the probability of overall survival, with censoring of data at 30 months. Kaplan-Meier estimates were used to calculate survival curves, and $P$ values were determined with the use of the log-rank test. Panel A shows the cumulative incidence of invasive aspergillosis in transplant recipients according to donor $+281 \mathrm{~A} / \mathrm{G}$ genotypes. The same plot also shows donor $+1449 \mathrm{~A} / \mathrm{G}$, since it displayed complete linkage disequilibrium $\left(r^{2}=1\right)$ with $+281 \mathrm{~A} / \mathrm{G}$ among cases of invasive aspergillosis. Panel B shows the cumulative incidence of invasive aspergillosis according to donor $+734 \mathrm{~A} / \mathrm{C}$ (D48A) genotypes. Panel $\mathrm{C}$ shows the cumulative incidence of invasive aspergillosis according to donor haplotypes hl/hl (A-C/A-C), hl/h2 (A-C/G-A), and $\mathrm{h} 2 / \mathrm{h} 2$ (G-A/G-A). The $+281 \mathrm{~A} / \mathrm{G}$ and $+1449 \mathrm{~A} / \mathrm{G}$ SNPs are in strong linkage disequilibrium and can be used interchangeably within haplotype block 3. Panel D shows the cumulative incidence of invasive aspergillosis according to donor haplotypes ( $h l / h l$, as well as $\mathrm{hl} / \mathrm{h} 2$ and h2/h2) and type of donor (HLA-matched related donor [MRD] or HLA-mismatched related donor [MMRD]). Haplotypes $\mathrm{hl} / \mathrm{hl}$ and $\mathrm{hl} / \mathrm{h} 2$ were combined in a single category, since they conferred a similar risk of invasive aspergillosis, as shown in Panel C. $\mathrm{P}=0.75$ for the test for interaction between the PTX3 haplotypes and the type of transplant. Panel $E$ shows overall survival according to donor PTX 3 variants. Shown are the results for patients who received transplants from MRDs with the hl/hl or hl/h2 haplotype versus patients who received transplants from other donors (MMRDs with the $\mathrm{hl} / \mathrm{hl}$ or hl/h2 haplotype and either MRDs or MMRDs with the h2/h2 haplotype).

among recipients after transplantation (Table S4 in the Supplementary Appendix), even though the cumulative incidence of infection among patients with A-C/A-C (block 1) donors was not significantly different from the incidence among patients with T-A/T-A donors and the cumulative incidence of infection among patients with T-G/T-G (block 2) donors was not significantly different from the incidence among patients with A-A/A-A donors (Fig. S2 in the Supplementary Appendix). However, the cumulative incidence of infection with transplants from G-A/G-A (hereafter referred to as $h 2 / h 2$ ) block 3 donors was significantly higher than that with transplants from A-C/A-C (h1/h1) donors and A-C/G-A (h1/h2) donors (37\% 


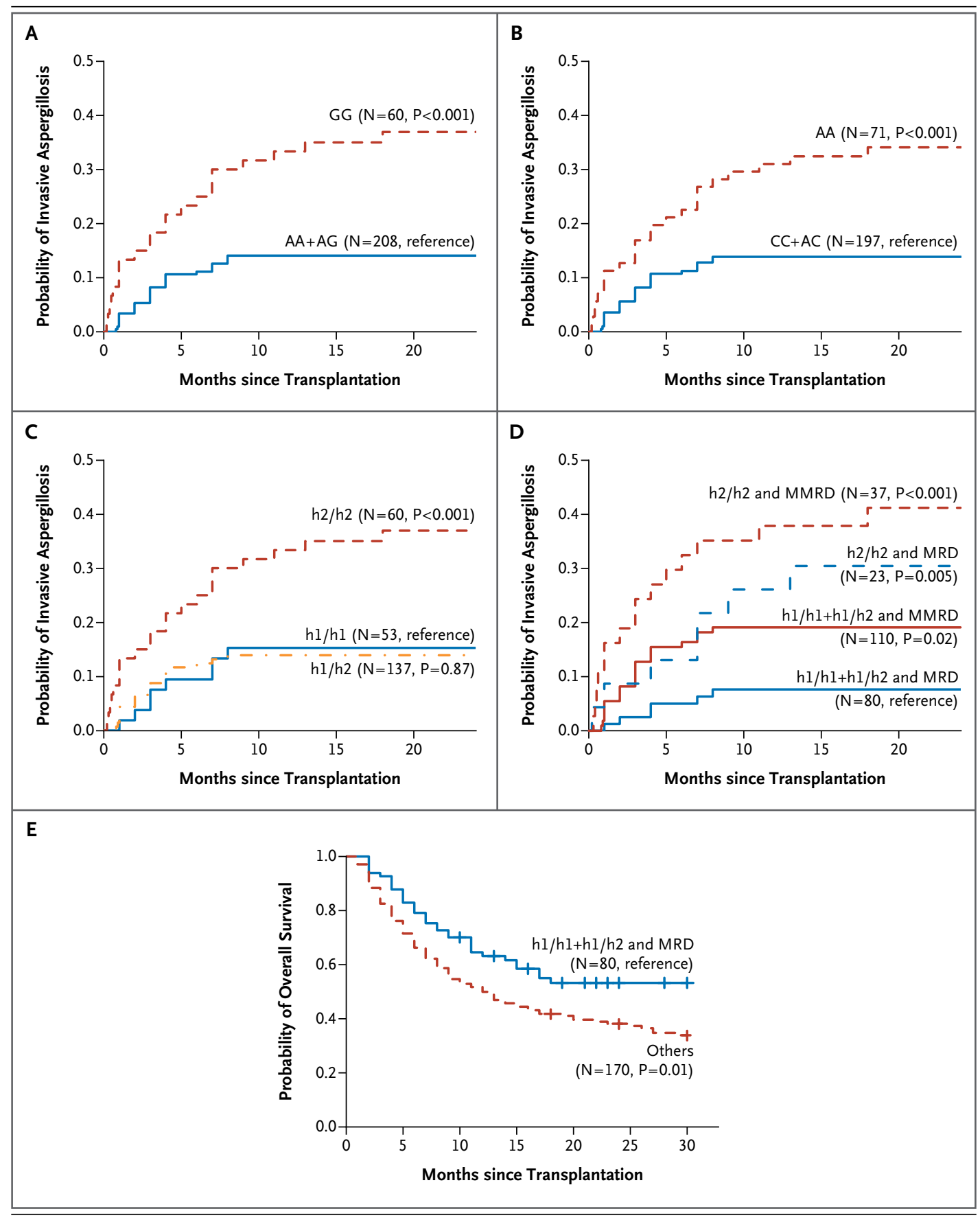

vs. $15 \%$ and $37 \%$ vs. $14 \%$, respectively; $\mathrm{P}<0.001$; adjusted $\mathrm{P}=0.001$ ); the cumulative incidence of infection with transplants from A-C/G-A (h1/h2) donors was similar to that with transplants from A-C/A-C (h1/h1) donors ( $\mathrm{P}=0.87$ ) (Fig. 1C).

With regard to rare haplotypes, an $18 \%$ incidence of invasive aspergillosis was observed among recipients of transplants from $\mathrm{A}-\mathrm{A} / \mathrm{A}-\mathrm{A}$ donors $(\mathrm{P}=0.85)$, whereas no cases were observed among recipients of transplants from donors with the A-C/A-A haplotype $(\mathrm{P}=0.31)$ (Fig. $\mathrm{S} 3$ in the Supplementary Appendix).

In the final multivariate SNP models, the adjusted hazard ratio for invasive aspergillosis 
with either donor genotype $+281 \mathrm{GG}$ or donor genotype $+1449 \mathrm{GG}$ was 2.92 (95\% confidence interval [CI], 1.69 to 5.05; $\mathrm{P}<0.001$ ), and the hazard ratio with donor genotype +734AA was 2.62 (95\% CI, 1.52 to $4.54 ; \mathrm{P}<0.001$ ) (Table 2). In the final haplotype model, the hazard ratio for invasive aspergillosis with $\mathrm{h} 2 / \mathrm{h} 2$ carriage was 3.08 (95\% CI, 1.47 to 6.44; $\mathrm{P}=0.003$ ). Risk estimates were similar when patients with "possible" (as opposed to "probable" or "proven") aspergillosis were excluded and when patients with invasive fungal disease other than invasive aspergillosis were included (Fig. S4 in the Supplementary Appendix).

The characteristics of the patients in the confirmation case-control study were similar to those of the patients in the initial study (Table S1 in the Supplementary Appendix), but the confirmation study included recipients of transplants from related donors and recipients of transplants from unrelated donors. Haplotype frequencies in the confirmation population were similar to those in the discovery population (Table S5 in the Supplementary Appendix). The significant association between donor haplotype $\mathrm{h} 2 / \mathrm{h} 2$ and the risk of invasive aspergillosis in the discovery study (T-cell-depleted grafts and absence of GVHD and its prophylaxis) was also observed in the confirmation study (T-cellrepleted grafts and presence of GVHD and use of prophylaxis) (adjusted odds ratio, 2.78; $95 \%$ CI, 1.22 to 8.93; $\mathrm{P}=0.03$ ) (Table 2) but not in a second, independent confirmation study involving a cohort of patients with prolonged neutropenia who had not undergone HSCT (Table S6 in the Supplementary Appendix).

\section{RISK OF INVASIVE ASPERGILLOSIS ACCORDING TO DONOR HAPLOTYPE AND HLA-MATCHING STATUS}

Given that donor PTX3 haplotypes and HLA profile are pretransplantation predictors of the risk of invasive aspergillosis, we stratified patients in the discovery cohort according to the type of donor (HLA-matched related donor vs. HLA-mismatched related donor) and haplotype (h2/h2 vs. h1/h1 or h1/h2). Among transplant recipients whose donors had the h1/h1 or h1/h2 haplotype, the cumulative incidence of invasive aspergillosis increased from $8 \%$ among recipients with matched related donors to $19 \%$ among those with mismatched related donors $(\mathrm{P}=0.02)$ (Fig. 1D). However, in the presence of the h2/h2 haplotype, the risk increased further, from $30 \%$ among recipients with matched related donors $(\mathrm{P}=0.005$ for the comparison with $h 1 / h 1$ or h1/h2 matched related donors) to $41 \%$ among those with mismatched related donors $(\mathrm{P}<0.001$ for the comparison with h1/h1 or h1/h2 matched related donors). In a multivariate analysis, the hazard ratio for invasive aspergillosis among transplant re-

Table 2. Multivariate Analysis of the Association of Donor PTX3 Variants with the Risk of Invasive Aspergillosis among Transplant Recipients in the Discovery and Confirmation Studies.*

\begin{tabular}{|c|c|c|c|c|}
\hline \multirow[t]{2}{*}{ Donor PTX3 Variant } & \multicolumn{2}{|c|}{ Discovery Study $(\mathrm{N}=268)$} & \multicolumn{2}{|c|}{ Confirmation Study $(\mathrm{N}=330)$} \\
\hline & $\begin{array}{c}\text { Adjusted Hazard Ratio } \\
(95 \% \mathrm{Cl}) \dagger\end{array}$ & P Value & $\begin{array}{c}\text { Adjusted Odds Ratio } \\
(95 \% \mathrm{Cl}) ‡\end{array}$ & P Value \\
\hline +281A/G SNP, GG genotype & $2.92(1.69-5.05)$ & $<0.001$ & $2.14(1.20-3.80)$ & 0.01 \\
\hline +734A/C SNP, AA genotype & $2.62(1.52-4.54)$ & $<0.001$ & $1.92(0.91-3.04)$ & 0.07 \\
\hline Haplotype h2/h2 & $3.08(1.47-6.44)$ & 0.003 & $2.78(1.22-8.93)$ & 0.03 \\
\hline
\end{tabular}

* $\mathrm{Cl}$ denotes confidence interval, and SNP single-nucleotide polymorphism. Multivariate analyses were based on the subdistribution regression model of Fine and Gray ${ }^{22}$ in the discovery study and on conditional logistic regression in the confirmation study. In the SNP model, the genetic variants were computed one at a time with the clinical covariates. The results for donor +281GG genotype are identical to those obtained for the +1449GG genotype because of their complete linkage disequilibrium among cases of invasive aspergillosis. The combination of genotypes AA and AG was the reference category for $+281 \mathrm{~A} / \mathrm{G}$, and the combination of genotypes CC and CA was the reference category for $+734 \mathrm{~A} / \mathrm{C}$. For the haplotype model, the reference category was $\mathrm{hl} / \mathrm{hl}$. Hazard ratios and odds ratios are for the presence versus the absence of the genotype or haplotype that confers a risk of invasive aspergillosis.

$\uparrow$ Hazard ratios have been adjusted for HLA-matching status, use or nonuse of total-body irradiation in the myeloablative conditioning, and antifungal prophylaxis (fluconazole or liposomal amphotericin B). The only clinical variable that remained significantly associated with invasive aspergillosis in the SNP and haplotype models was receipt of a transplant from an HLA-mismatched relative (+281A/G: hazard ratio, 1.94; $95 \% \mathrm{Cl}, 1.07$ to $3.52 ; \mathrm{P}=0.03$; $+734 \mathrm{~A} / \mathrm{C}$ : hazard ratio, $1.88 ; 95 \% \mathrm{Cl}, 1.04$ to $3.42 ; \mathrm{P}=0.04$; haplotype $\mathrm{h} 2 / \mathrm{h} 2$ : hazard ratio, $1.95 ; 95 \% \mathrm{Cl}, 1.06$ to $3.58 ; \mathrm{P}=0.03$ ).

$\dagger$ Odds ratios have been adjusted for HLA-matching status and use or nonuse of total-body irradiation in the myeloablative conditioning regimen. 
cipients with $\mathrm{h} 1 / \mathrm{h} 1$ or $\mathrm{h} 1 / \mathrm{h} 2$ mismatched related donors versus those with matched related donors was 2.46 (95\% CI, 1.10 to 5.50; $\mathrm{P}=0.03$ ). Among transplant recipients with $\mathrm{h} 2 / \mathrm{h} 2$ donors, the hazard ratio, with h1/h1 or h1/h2 matched related donors as the reference group, was further increased, from 3.96 (95\% CI, 1.49 to 10.50; $\mathrm{P}=0.006$ ) among recipients with matched related donors to 6.02 (95\% CI, 2.58 to 14.00 ; $\mathrm{P}<0.001)$ among those with mismatched related donors. Accordingly, the probability of survival at 30 months after transplantation decreased from $63 \%$ among patients who received transplants from matched related donors with haplotype h1/h1 or h1/h2 to $37 \%$ among patients who received transplants from either h1/h1 or h1/h2 mismatched related donors or from $\mathrm{h} 2 / \mathrm{h} 2$ matched or mismatched related donors $(\mathrm{P}=0.01)$ (Fig. 1E).

\section{EFFECT OF THE +734A/C SNP ON PTX3 MESSENGER RNA FOLDING}

To determine the molecular consequences of the SNPs in PTX3 haplotype block 3, we examined the pathogenic potential of each SNP in terms of protein function. We first compared the amino acid sequence of annotated PTX3 proteins among several mammals to determine whether the $+734 \mathrm{~A} / \mathrm{C}$ (D48A) mutation was occurring in a phylogenetically conserved site. Alignment of PTX3 sequences confirmed that the D48A substitution leading to an alanine residue in humans lies in an evolutionarily conserved region within the mammalian lineage, since all other species carried an aspartic acid residue instead (Fig. S5 in the Supplementary Appendix). Even though this mutation affected a conserved site, it was nonetheless predicted in silico as benign with respect to protein folding and structural stability. More important, when we looked at models of the effect of the $+734 \mathrm{~A} / \mathrm{C}$ SNP on PTX3 messenger RNA (mRNA) folding, we found it to be severely affected by the nucleotide substitution (Fig. S6 in the Supplementary Appendix), indicating altered mRNA stability. No differences in splicing were predicted for intronic SNPs (Table S7 in the Supplementary Appendix).

\section{DECREASED EXPRESSION OF PTX3 WITH THE H2/H2 HAPLOTYPE}

To assess the functional consequences of $h 2 / h 2$, we compared PTX3 expression in vivo in the lungs of transplant recipients with invasive aspergillosis and in those without invasive aspergillosis, as well as in vitro on purified cells. We found higher levels of PTX3 in bronchoalveolar-lavage fluid, but not in plasma (Fig. S7 in the Supplementary Appendix), from patients with invasive aspergillosis than in patients without invasive aspergillosis (Fig. 2A). More important, median ( \pm SD) PTX3 levels in bronchoalveolar-lavage fluid differed significantly according to donor haplotype both for patients with invasive aspergillosis $(2.50 \pm 0.73 \mathrm{ng}$ per milliliter for h1/h1 [14 patients] vs. $1.36 \pm 0.14 \mathrm{ng}$ per milliliter for h2/h2 [15 patients]; $\mathrm{P}<0.001$ ) and for those without invasive aspergillosis $(0.83 \pm 0.06$ ng per milliliter for h1/h1 [21 patients] vs. $0.47 \pm 0.03$ ng per milliliter for h2/h2 [21 patients]; $\mathrm{P}<0.001)$. Further supporting these findings, immunofluorescence analysis of lung-biopsy specimens from patients with invasive aspergillosis revealed decreased positivity for PTX3 in lung tissue from transplant recipients with $\mathrm{h} 2 / \mathrm{h} 2$ donors (4 patients) as compared with transplant recipients with h1/h1 donors (5 patients) (Fig. 2B).

We performed in vitro assessment of mRNA and protein expression in purified peripheralblood neutrophils, one major cell type expressing PTX3. Mature neutrophils do not contain detectable levels of PTX3 mRNA. ${ }^{7}$ In contrast, neutrophil precursors (most notably promyelocytes, as well as myelocytes plus metamyelocytes) express PTX3 transcript. ${ }^{7}$ The h2/h2 haplotype was correlated with lower levels of PTX3 mRNA in $\mathrm{h} 2 / \mathrm{h} 2$ precursors than in $\mathrm{h} 1 / \mathrm{h} 1$ precursors (3 donors for each haplotype) (Fig. 2C). Analysis of intracellular protein levels revealed that PTX3 was constitutively present in resting neutrophils, although protein levels were markedly lower in $\mathrm{h} 2 / \mathrm{h} 2$ neutrophils (in 14 patients) than in $\mathrm{h} 1 / \mathrm{h} 1$ neutrophils (in 12 patients) (Fig. 2D). Defective expression of intracellular PTX3 in h2/h2 neutrophils as compared with h1/h1 neutrophils (8 patients for each haplotype) was confirmed by means of Western blotting (Fig. 2E) and immunofluorescence (Fig. 2F).

\section{ANTIFUNGAL CAPACITY OF NEUTROPHILS AND THE H2/H2 HAPLOTYPE}

To ascertain the functional consequences of the h2/h2 haplotype on antifungal effector mechanisms, we investigated the effector capacity of neutrophils bearing distinct haplotypes. Neutrophils from $\mathrm{h} 2 / \mathrm{h} 2$ patients showed a significantly impaired ability to phagocytose conidia of A. fumig- 


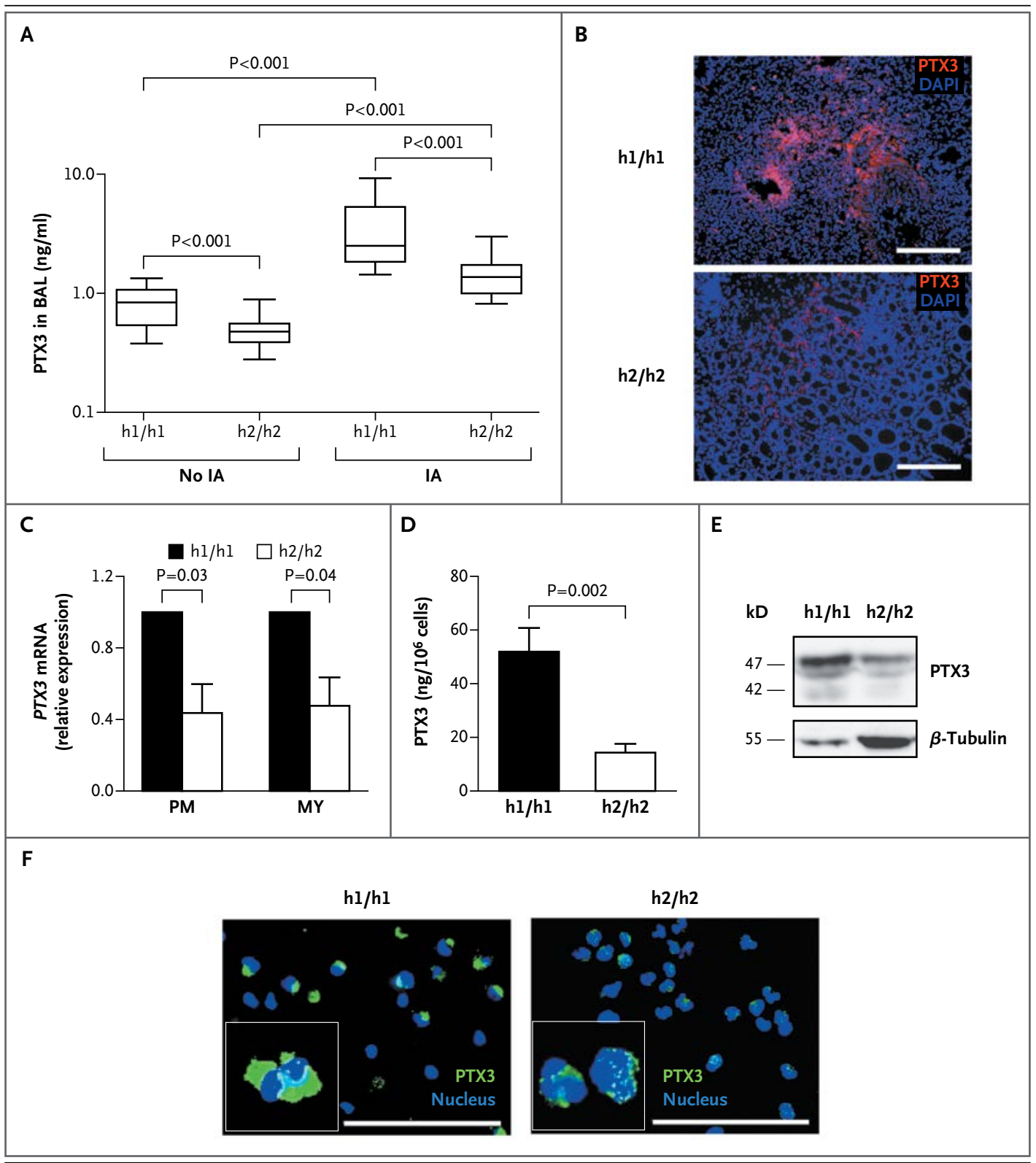

atus, with a 40-percentage-point reduction, as compared with h1/h1 cells (Fig. 3A). Moreover, h2/h2 neutrophils also showed a 20-percentagepoint decrease in their conidiocidal activity in general, as compared with h1/h1 cells (Fig. 3B). Since PTX3 haplotype block 3 underlies two distinct forms of PTX3 on the basis of the D48A substitution (48D and 48A), we assessed whether the overall defective antifungal activity displayed by h2/h2 neutrophils was linked to an altered ability of either form to bind conidia. However, both forms efficiently bound to the fungus, as assessed with the use of fluorescence-labeled PTX3 (Fig. 3C).

Given that exogenous PTX3 restores antifungal effector mechanisms in PTX3-deficient cells, $, 3,8$ we analyzed whether the addition of PTX3 reversed the antifungal effector deficiencies observed in h2/h2 neutrophils. The addition of either form of PTX3 significantly enhanced phagocytosis of conidia by $\mathrm{h} 2 / \mathrm{h} 2$ neutrophils (63\% for $48 \mathrm{D}$, and $57 \%$ for $48 \mathrm{~A}$ ) and $\mathrm{h} 1 / \mathrm{h} 1$ neu- 
Figure 2 (facing page). Effect of the h2/h2 Haplotype on PTX3 Expression.

Panel A shows PTX3 levels in samples of bronchoalveolar-lavage fluid (BAL) from patients with proven or probable invasive aspergillosis (IA) and those without IA whose donors had hl/hl (A-C/A-C) (14 patients with IA and 21 without IA) or h2/h2 (G-A/G-A) (15 patients with $I A$ and 21 without IA). Shown are median $( \pm$ SD) levels (horizontal lines within the boxes) and interquartile ranges (lines above and below the boxes), assessed in duplicate. $P$ values were calculated with the use of the two-tailed Mann-Whitney rank-sum test. Panel B shows immunofluorescence staining of lungbiopsy specimens with antihuman PTX 3 as the primary antibody and antirabbit tetramethylrhodamine isothiocyanate as the secondary antibody. DAPI $(4,6$-diamidino2-phenylindole) was used to counterstain tissues. Images are representative of at least four samples for each haplotype assessed in two distinct preparations. Scale bars indicate $100 \mu \mathrm{m}$. Panel $\mathrm{C}$ shows analysis of PTX 3 mRNA expression in promyelocytes (PM) and myelocytes plus metamyelocytes (MY). Shown is the mean $( \pm \mathrm{SD})$ messenger RNA (mRNA) level in three bone marrow donors for each haplotype, assessed in triplicate. Panel D shows PTX 3 levels in neutrophils as assessed by enzyme-linked immunosorbent assay. Shown is the mean $( \pm S D)$ protein level in at least 12 patients for each donor haplotype, assessed in duplicate. In Panels $C$ and $D, P$ values were calculated with the use of an unpaired Student's t-test with Bonferroni's adjustment. Panel E shows Western blot analysis of PTX3 expression in neutrophils. Blots of cell lysates were incubated with a polyclonal antibody against PTX3, and normalization was performed with an antihuman $\beta$-tubulin antibody. Panel $\mathrm{F}$ shows immunofluorescence imaging of PTX 3 in neutrophils. Neutrophils were incubated with a primary antibody against PTX3, followed by a secondary fluorescein isothiocyanate (FITC)-conjugated antirabbit IgG antibody. Nuclei were counterstained with DAPI. Scale bars indicate $100 \mu \mathrm{m}$. The findings in Panels $E$ and $F$ are representative of those in at least eight patients tested for each haplotype combination, assessed in triplicate.

trophils ( $80 \%$ for $48 \mathrm{D}$ and $73 \%$ for $48 \mathrm{~A}$ ) (Fig. 3D). The phagocytic activity of $\mathrm{h} 2 / \mathrm{h} 2$ neutrophils was further improved on preopsonization of conidia with either form of PTX3, since these cells were able to internalize conidia even more efficiently (82\% for $48 \mathrm{D}$, and $80 \%$ for $48 \mathrm{~A}$ ). Similarly, the conidiocidal activity of h2/h2 neutrophils was restored to $62 \%$ by the direct addition of $48 \mathrm{D}$ and to $50 \%$ by the direct addition of $48 \mathrm{~A}$, as well as to $60 \%$ after preopsonization of conidia with $48 \mathrm{D}$ and to $57 \%$ after preopsonization of conidia with $48 \mathrm{~A}$ (Fig. 3E).
DISCUSSION

In this study, we found an association between donor h2/h2 haplotype in PTX3 and invasive aspergillosis in patients undergoing HSCT, a finding that supports the nonredundant role of PTX3 in host defense against A. fumigatus. ${ }^{3,4,7}$ More important, the increased risk of infection was observed regardless of the HLA-matching status of the donor, T-cell manipulation, and acute GVHD and prophylaxis, further sustaining a potentially independent contribution of PTX3 variants to the development and outcome of invasive aspergillosis in the context of various strategies for modulating lymphocyte function.

PTX3 SNPs have been shown to modify the risk of pulmonary tuberculosis ${ }^{23}$ and colonization by Pseudomonas aeruginosa in patients with cystic fibrosis. ${ }^{24}$ Furthermore, these SNPs were found to alter blood levels of the PTX3 protein in Ghanaian women ${ }^{25}$ and in lung-transplant recipients with primary graft dysfunction. ${ }^{26}$ In our study, the $\mathrm{h} 2 / \mathrm{h} 2$ haplotype was consistently associated with a defect in PTX3 expression in bronchoalveolar-lavage fluid, lung-biopsy specimens, and innate immune cells. This association was not confirmed systemically, a finding that suggests a requirement for the presence of PTX3 on the infected lung, where it opsonizes the fungus and helps to resolve infection.

Although neutropenia is an important risk factor for opportunistic fungal infections, most cases of aspergillosis in patients who have undergone allogeneic HSCT occur a few months after transplantation, when neutrophil levels have returned to the normal range, suggesting that neutrophils, which express PTX3 and are required for resistance to A. fumigatus, ${ }^{7}$ may be particularly relevant for the observed susceptibility phenotype. Indeed, PTX3 SNPs were not associated with invasive aspergillosis in patients with prolonged neutropenia who did not undergo transplantation, supporting the importance of the PTX3 defect in neutrophils. Because PTX3 has a role as an opsonin, limiting the amount of PTX3 available to bind the fungus most likely restrains the efficiency of phagocytosis and fungal clearance, as was observed for $\mathrm{h} 2 / \mathrm{h} 2$ neutrophils. The exogenous addition of PTX3 to PTX3-deficient neutrophils reversed the functional deficit, confirming that the innate antifungal mechanisms 


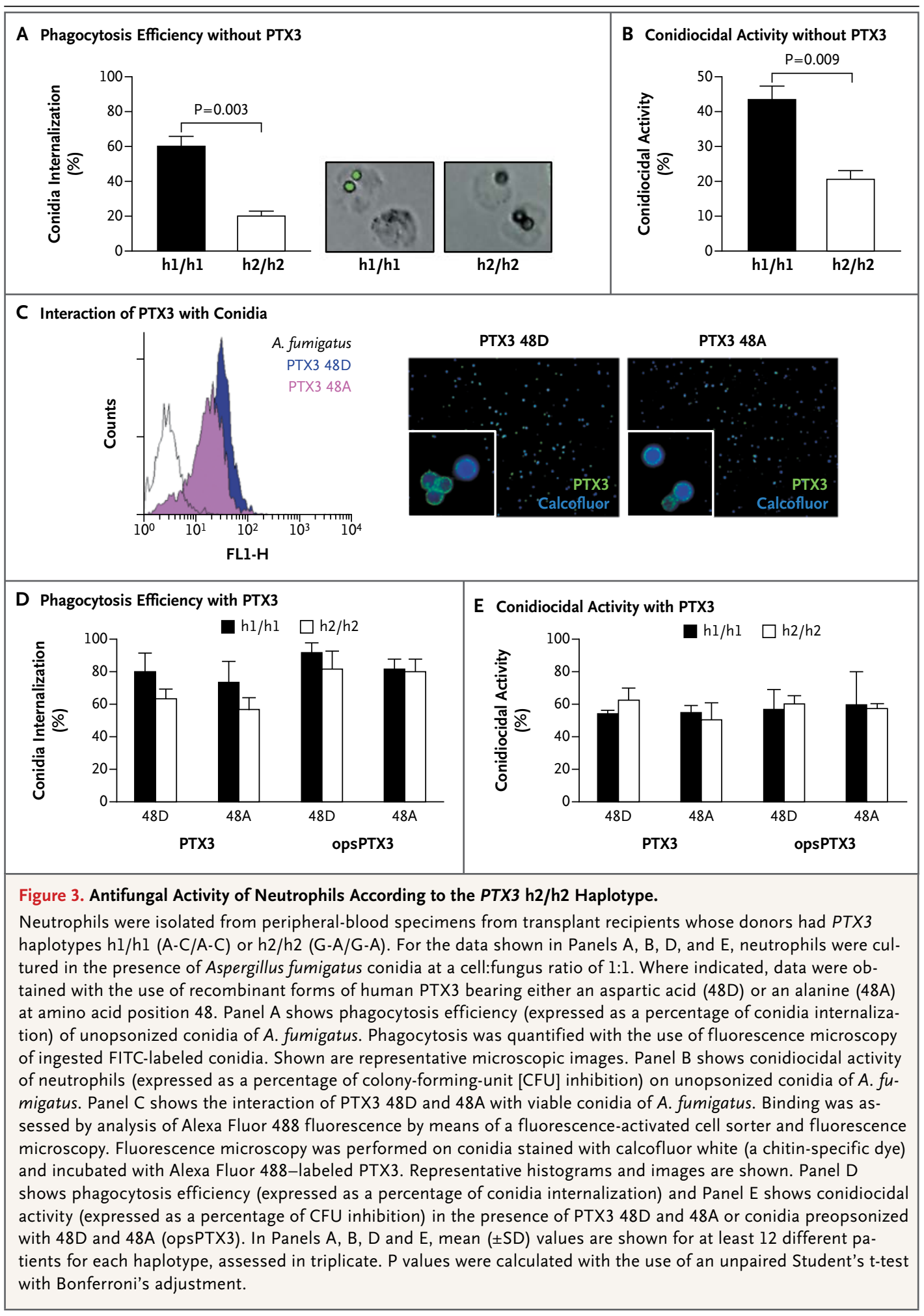


of these cells are compromised by a lack of sufficient PTX3 to effectively counter the fungus. Our observation that both 48A and 48D forms of PTX3 bound conidia efficiently further supports a deficiency of PTX3 in quantity, not in quality. It is also possible that PTX3 deficiency adversely affects antifungal mechanisms mediated by other innate immune receptors ${ }^{27}$ or leukocyte recruitment in the lungs. ${ }^{28}$

Whatever the mechanism (or mechanisms), the absence of an association between genetic variants in transplant recipients and invasive aspergillosis suggests that epithelial PTX3 has a smaller role, if any, in the immune response to the fungus. PTX3 expression was not detectable in epithelial cells stimulated with A. fumigatus conidia, ${ }^{3}$ even though its induction by proinflammatory stimuli in human lung epithelial cells has been documented. ${ }^{29}$ Exogenous Ptx3 has been found to drive innate antifungal resistance of epithelial cells through defensins and enhancement of fungal phagocytosis, ${ }^{8}$ suggesting that myeloid PTX3 may nonetheless play an important part in regulating epithelial antifungal mechanisms. It will be important to determine whether PTX3 deficiency also affects the initiation and skewing of adaptive immune responses to the fungus, as shown in Ptx3-deficient murine dendritic cells. ${ }^{3}$

Mechanistically, the decreased expression of PTX3 transcript in neutrophil precursors is consistent with the predicted alterations in the
mRNA secondary structure, suggesting that the $+734 \mathrm{~A} / \mathrm{C}$ nucleotide change most likely affects regulation of PTX3 expression. Also, a contribution of the intronic SNPs from the h2/h2 haplotype or other SNPs in the $5^{\prime}$ region to the observed phenotype cannot be ruled out. However, even though no alterations in PTX3 structure were identified as a consequence of the D48A substitution, damage to its electrostatic potential or interactions with other proteins also cannot be ruled out. The fact that the adjacent positions 47 and 49 encode cysteine residues involved in the formation of PTX3 protein complex octamers $^{30}$ supports this possibility.

In conclusion, we found that genetic deficiency of PTX3 affects the antifungal function of neutrophils. This deficiency may increase susceptibility to invasive aspergillosis in patients undergoing HSCT.

\footnotetext{
Supported by grants from the European Society of Clinical Microbiology and Infectious Diseases (ESCMID) (to Dr. Carvalho); the German Ministry for Education and Science (03Z2JN21, to Dr. Kurzai); the European Commission (FP7-HEALTH-2009-260338, to Dr. Romani; FP7-HEALTH-2011-280873, to Dr. Mantovani), the European Research Council (ERC-2008-AdG-233417, to Dr. Mantovani; ERC-2011-AdG-293714, to Dr. Romani), Associazione Italiana per la Ricerca sul Cancro (99629, to Dr. Mantovani); and Fundação para a Ciência e Tecnologia, Portugal (SFRH/BPD/ 46292/2008, to Dr. Carvalho; SFRH/BD/65962/2009, to Dr. Cunha; and SFRH/BPD/70783/2010, to Dr. Almeida).

Disclosure forms provided by the authors are available with the full text of this article at NEJM.org.

We thank Rhian Gwilliam (LGC Genomics) for advice on SNP selection and assay design.
}

APPENDIX

The authors' affiliations are as follows: Departments of Experimental Medicine and Biochemical Sciences (C.C., A.V., L.R., A.C.) and Clinical and Experimental Medicine (A.S.B.), University of Perugia, Perugia, the Department of Clinical and Experimental Medicine, University of Parma, Parma (F.A.), Trapianto di Cellule Staminali-Ematologia 2 (A. Busca) and S.C. Microbiologia (A. Barbui), A.O. Città della Salute e della Scienza, Torino, Department of Inflammation and Immunology, Humanitas Clinical and Research Center, Rozzano (A.I., E.B., A.M.), Department of Translational Medicine, University of Milan, Milan (A.M.), Department of Medical and Surgical Sciences, University of Modena and Reggio Emilia, AOU Policlinico, Modena (L. Potenza, M.L.), Istituto di Ematologia, Università Cattolica del S. Cuore, Rome (M.C., L. Pagano), and Sigma-tau Industrie Farmaceutiche Riunite, Pomezia (G.S.) — all in Italy; Hospital de Santa Maria, University of Lisbon, Lisbon (J.F.L., P.S.S.), Institute for Molecular and Cell Biology, Universidade do Porto, Porto (B.A.), Life and Health Sciences Research Institute (ICVS), School of Health Sciences, University of Minho, Braga (F.R., A.C.), and ICVS/3B's-PT Government Associate Laboratory, Braga/Guimarães (F.R., A.C.) — all in Portugal; Septomics Research Center, FriedrichSchiller-University and Leibniz-Institute for Natural Products Research and Infection Biology, Hans-Knoell-Institute, Jena (O.K.), Department of Hematology and Oncology, University of Regensburg, Regensburg (M.G.), and Medizinische Klinik und Poliklinik II, Universitätsklinikum Würzburg, Würzburg (J.L.) — all in Germany; and the Department of Hematology, Universitaire Ziekenhuizen Leuven, Campus Gasthuisberg, Leuven, Belgium (J.A.M.).

\section{REFERENCES}

1. Garlanda C, Bottazzi B, Bastone A Mantovani A. Pentraxins at the crossroads between innate immunity, inflammation, matrix deposition, and female fertility. Annu Rev Immunol 2005;23:337-66.

2. Souza DG, Soares AC, Pinho V, et al.
Increased mortality and inflammation in tumor necrosis factor-stimulated gene-14 transgenic mice after ischemia and reperfusion injury. Am J Pathol 2002;160:175565.

3. Garlanda C, Hirsch E, Bozza S, et al.
Non-redundant role of the long pentraxin PTX3 in anti-fungal innate immune response. Nature 2002;420:182-6.

4. Moalli F, Doni A, Deban L, et al. Role of complement and Fcgamma receptors in the protective activity of the long pen- 
traxin PTX3 against Aspergillus fumigatus. Blood 2010;116:5170-80.

5. Tierney L, Linde J, Müller S, et al. An interspecies regulatory network inferred from simultaneous RNA-seq of Candida albicans invading innate immune cells. Front Microbiol 2012;3:85.

6. Diniz SN, Nomizo R, Cisalpino PS, et al. PTX3 function as an opsonin for the dectin-1-dependent internalization of zymosan by macrophages. J Leukoc Biol 2004;75:649-56.

7. Jaillon S, Peri G, Delneste Y, et al. The humoral pattern recognition receptor PTX3 is stored in neutrophil granules and localizes in extracellular traps. J Exp Med 2007;204:793-804.

8. D’Angelo C, De Luca A, Zelante T, et al. Exogenous pentraxin 3 restores antifungal resistance and restrains inflammation in murine chronic granulomatous disease. J Immunol 2009;183:4609-18.

9. Gaziano R, Bozza S, Bellocchio S, et al. Anti-Aspergillus fumigatus efficacy of pentraxin 3 alone and in combination with antifungals. Antimicrob Agents Chemother 2004;48:4414-21.

10. Lo Giudice P, Campo S, De Santis R, Salvatori G. Effect of PTX3 and voriconazole combination in a rat model of invasive pulmonary aspergillosis. Antimicrob Agents Chemother 2012;56:6400-2.

11. Salvatori G, Campo S. Current understanding of PTX3 protective activity on Aspergillus fumigatus infection. Med Mycol 2012;50:225-33.

12. Kontoyiannis DP, Marr KA, Park BJ, et al. Prospective surveillance for invasive fungal infections in hematopoietic stem cell transplant recipients, 2001-2006: overview of the Transplant-Associated Infection Surveillance Network (TRANSNET) Database. Clin Infect Dis 2010;50:1091-100. 13. Pagano L, Caira M, Nosari A, et al. Fungal infections in recipients of hemato- poietic stem cell transplants: results of the SEIFEM B-2004 study — Sorveglianza Epidemiologica Infezioni Fungine Nelle Emopatie Maligne. Clin Infect Dis 2007; 45:1161-70.

14. Pappas PG, Alexander BD, Andes DR, et al. Invasive fungal infections among organ transplant recipients: results of the Transplant-Associated Infection Surveillance Network (TRANSNET). Clin Infect Dis 2010;50:1101-11.

15. Di Ianni M, Falzetti F, Carotti A, et al. Tregs prevent GVHD and promote immune reconstitution in HLA-haploidentical transplantation. Blood 2011;117:3921-8.

16. De Pauw B, Walsh TJ, Donnelly JP, et al. Revised definitions of invasive fungal disease from the European Organization for Research and Treatment of Cancer/ Invasive Fungal Infections Cooperative Group and the National Institute of Allergy and Infectious Diseases Mycoses Study Group (EORTC/MSG) Consensus Group. Clin Infect Dis 2008;46:1813-21.

17. Aversa F, Tabilio A, Velardi A, et al Treatment of high-risk acute leukemia with T-cell-depleted stem cells from related donors with one fully mismatched HLA haplotype. N Engl J Med 1998;339: 1186-93.

18. Ljungman P, Griffiths P, Paya C. Definitions of cytomegalovirus infection and disease in transplant recipients. Clin Infect Dis 2002;34:1094-7.

19. Przepiorka D, Weisdorf D, Martin P, et al. 1994 Consensus Conference on Acute GVHD Grading. Bone Marrow Transplant 1995;15:825-8.

20. Gray RJ. A class of K-sample tests for comparing the cumulative incidence of a competing risk. Ann Stat 1988;16:1141-54. 21. Scrucca L, Santucci A, Aversa F. Competing risk analysis using $\mathrm{R}$ : an easy guide for clinicians. Bone Marrow Transplant 2007;40:381-7.
22. Fine JP, Gray RJ. A proportional hazards model for the subdistribution of a competing risk. J Am Stat Assoc 1999; 94:496-509.

23. Olesen R, Wejse C, Velez DR, et al. DC-SIGN (CD209), pentraxin 3 and vitamin D receptor gene variants associate with pulmonary tuberculosis risk in West Africans. Genes Immun 2007;8:456-67. 24. Chiarini M, Sabelli C, Melotti P, et al. PTX3 genetic variations affect the risk of Pseudomonas aeruginosa airway colonization in cystic fibrosis patients. Genes Immun 2010;11:665-70.

25. May L, Kuningas M, van Bodegom D, et al. Genetic variation in pentraxin (PTX) 3 gene associates with PTX3 production and fertility in women. Biol Reprod 2010; 82:299-304.

26. Diamond JM, Meyer NJ, Feng R, et al. Variation in PTX3 is associated with primary graft dysfunction after lung transplantation. Am J Respir Crit Care Med 2012;186:546-52.

27. Bozza S, Bistoni F, Gaziano R, et al. Pentraxin 3 protects from MCMV infection and reactivation through TLR sensing pathways leading to IRF3 activation. Blood 2006;108:3387-96.

28. Deban L, Russo RC, Sironi M, et al. Regulation of leukocyte recruitment by the long pentraxin PTX3. Nat Immunol 2010;11:328-34

29. Han B, Mura M, Andrade CF, et al. TNFalpha-induced long pentraxin PTX3 expression in human lung epithelial cells via JNK. J Immunol 2005;175:8303-11. 30. Inforzato A, Rivieccio $\mathrm{V}$, Morreale AP, et al. Structural characterization of PTX3 disulfide bond network and its multimeric status in cumulus matrix organization. J Biol Chem 2008;283:10147-61.

Copyright (@) 2014 Massachusetts Medical Society.

AN NEJM APP FOR IPHONE

The NEJM Image Challenge app brings a popular online feature to the smartphone. Optimized for viewing on the iPhone and iPod Touch, the Image Challenge app lets you test your diagnostic skills anytime, anywhere. The Image Challenge app randomly selects from 300 challenging clinical photos published in NEJM, with a new image added each week. View an image, choose your answer, get immediate feedback, and see how others answered.

The Image Challenge app is available at the iTunes App Store. 